\title{
Supporting Information \\ Synthesis of novel metal sulfide-polymer composite microspheres exhibiting patterned surface structures
}

Chaoliang Bai, Yu Fang, Ying Zhang, and Beibei Chen

School of Chemistry and Materials Science, Shaanxi Normal University, Xi'an 710062, P. R. China. Fax: 008629 5307025; Tel: 008629 5307534(L); E-mail: yfang@ snnu.edu.cn.

1. The surface of the microgels in swelled state is smooth (Fig. 1a, b). The patterned surface structures of the PbS-PNIPAM-MAA composite microspheres are different from those of either $\mathrm{ZnS}$ -
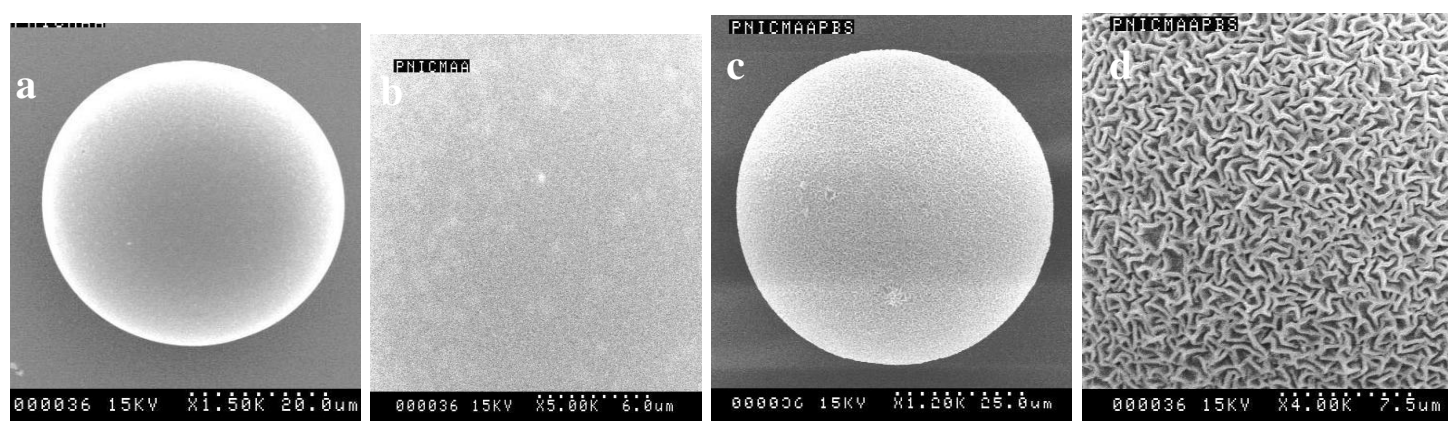

Fig. 1 SEM images of PNIPAM-MAA microgels (a), PbS-PNIPAM-MAA composite microspheres (c) and high magnification images of the surfaces $(b, d)$. The content of MAA in the template is $20 \%$.

PNIPAM-MAA or CdS-PNIPAM-MAA, indicating again the surface pattern is dependent upon the nature of the precipitate.

2. To gain insight into the phase of the precipitation, the composite was air dried, and analyzed with a Japan Rigaku D/MAX-III X-ray diffractometer,

which was equipped with graphite high-intensity $\mathrm{Cu}-\mathrm{K} \alpha$ radiation. The $\mathrm{X}$-ray diffraction spectra were taken for $2 \theta$ angles from $5^{\circ}$ to $60^{\circ}$ at a scan rate of $0.02 \% \mathrm{~min}$. Accelerating voltage and electric current are $35 \mathrm{kV}$ and $40 \mathrm{~mA}$, respectively. The XRD patterns of the metal sulfides-PNIPAM-MAA composites are shown in Fig. 2. As that expected, no sharp peaks in the spectrum of the microgels indicate that the microgels are in amorphous state. In contrast, the profile of the organic-inorganic composites

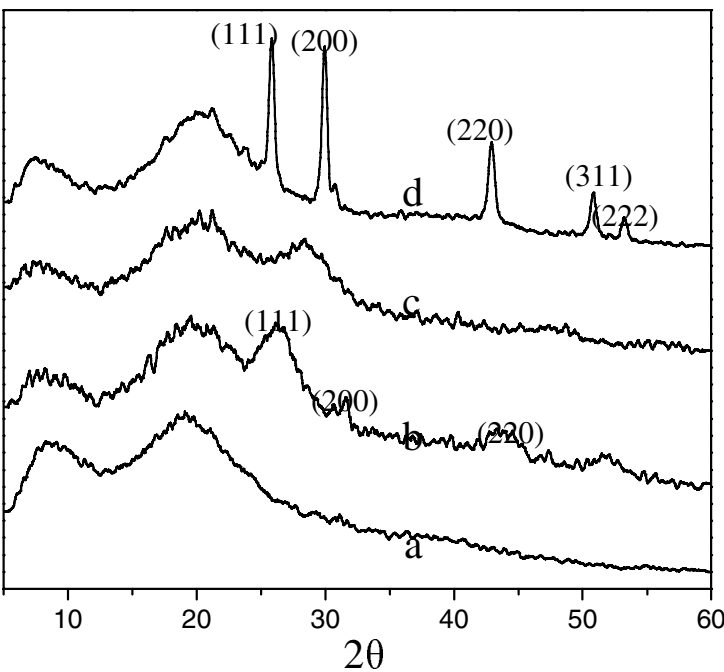

Fig. 2 XRD patterns (a) PNIPAM-MAA microgels; (b) CdS-PNIPAM-MAA; (c) ZnS-PNIPAM-MAA; (d) PbS-PNIPAM-MAA contains a number of new sharp (broad) peaks showing that some crystalline materials are present within the samples. 